\title{
Pulsatile Aortic Pressure-Flow Analysis using Fractional Calculus for Minimally-invasive Applications
}

\author{
Glen Atlas ${ }^{1,2}$, Sunil Dhar ${ }^{3}$, John K-J. Li ${ }^{4,5}$ \\ ${ }^{1}$ Rutgers New Jersey Medical School, Dept. of Anesthesiology \\ Newark, New Jersey, USA, 07039 \\ atlasgm@njms.rutgers.edu \\ ${ }^{2}$ Stevens Institute of Technology, Dept. of Chemistry, Chemical Biology, and Biomedical Engineering \\ Hoboken, New Jersey, USA, 07030 \\ ${ }^{3}$ New Jersey Institute of Technology, Dept. of Mathematical Sciences \\ Newark, New Jersey, USA, 07102 \\ ${ }^{4}$ Rutgers University, Department of Biomedical Engineering \\ Piscataway, New Jersey 08854, USA \\ ${ }^{5}$ College of Biomedical Engineering and Instrument Science \\ Zhejiang University \\ Hangzhou, China
}

\begin{abstract}
Time-dependent pulsatile pressure and flow waveforms in the aorta carry with them considerable information regarding the underlying dynamic behavior of the cardiovascular system. The aortic pressure-flow relationship has traditionally been described using integer calculus. As such, periodicity and linear system assumptions are necessarily imposed to extract hemodynamic information. We introduce the use of fractional calculus (FC) to relate minimally-invasive measurements, of the velocity of aortic blood flow with an esophageal Doppler monitor, to the derived aortic pressure. The basis for this research is a Taylor series model of the velocity of aortic blood flow with subsequent term-by-term fractional integration as well as fractional differentiation. These results demonstrate that this FC approach could potentially generate the aortic pressure waveform throughout systole. Further studies of its first derivative, or the time rate of pressure change, $\frac{d P}{d t}$, may also allow its maximal value, $\max \left(\frac{d P}{d t}\right)$, to be computed for use as an index of left ventricle contractility when noninvasive ultrasound Doppler flow velocity is available in the clinical setting.
\end{abstract}

Keywords: Fractional calculus, Aortic blood flow, Esophageal Doppler monitor, Differintegral, Differintegration.

(C) Copyright 2014 Authors - This is an Open Access article published under the Creative Commons Attribution License terms (http://creativecommons.org/licenses/by/3.0). Unrestricted use, distribution, and reproduction in any medium are permitted, provided the original work is properly cited.

Disclaimer: Presented in part at the International Conference on Biomedical Engineering and Systems, Prague, Czech Republic, August 14-15, 2014 and on the NJIT Center for Applied Mathematics and Statistics website: http://m.njit.edu/CAMS/Technical_Reports/CAMS13_14/repor t1314-18.pdf

\section{Introduction}

Hemodynamic diagnosis and clinical management often focus on blood pressure and flow measurements and subsequent analysis of their temporal relationship [1]. Routine catheterization can readily provide left ventricular pressure and aortic pressure, as well as cardiac output, from thermodilution. However, beat-tobeat flow measurements are less common. On the other hand, ultrasound Doppler echocardiography can provide images of cardiac and large vessel structures as well as blood flow velocity. Nonetheless, the aortic blood pressure waveform cannot be obtained.

Noninvasive or minimally-invasive approaches are preferred methods for routine clinical diagnosis and follow-up. Tonometer-based measurements, of blood 
pressure overlying the carotid artery, have been common [2], but are much less frequently used in conjunction with Doppler ultrasound in the clinical setting. It is possible to derive central aortic pressure when the Doppler ultrasound aortic flow velocity is known [3], [4]. These latter traditional hemodynamic models are typically based upon a linear second-order system utilizing the acceleration, velocity, and displacement of blood flow [5]. By convention, acceleration is defined as the first derivative of velocity, with respect to time, whereas displacement is its indefinite integral. In contradistinction, fractional calculus (FC) is based upon both integer and non-integer differentiation as well as integer and non-integer integration [6], [7].

\section{Related Work}

FC, although three centuries-old, has recently found applications in the analysis of biological systems. For instance, Djordjevic et al. [8] developed a rheological model of airway smooth muscle cells using a method incorporating FC and a least-squares data fitting technique. They showed that FC could be effectively utilized to account for a weak power law frequency dependence of cell rheological behavior. This effect could not be explained with traditional viscoelastic theory. Recently, an FC dynamic model has been applied to generate electrocardiogram (ECG) signals based upon oscillations and a global optimization scheme. This technique subsequently generates a realistic time series, of the ECG signal, and may find potential applications in modeling abnormal and irregular patterns of cardiac conduction [9].

We have considerable experience with the use of esophageal Doppler monitor (EDM) for minimallyinvasive measurement of the aortic flow velocity waveform [5]. This paper provides the first such application of FC to minimally-invasive hemodynamic studies and demonstrates how FC-based modelling could be effectively utilized in understanding the aortic pressure-flow relationship during systole.

\section{Methods}

\subsection{Fractional Calculus}

To cognize this application of $\mathrm{FC}$, traditional integer differentiation is first examined for a power function of time:

$$
f(t)=t^{m}
$$

$$
\begin{aligned}
& f^{\prime}(t)=m t^{(m-1)} . \\
& f^{\prime \prime}(t)=m(m-1) t^{(m-2)} . \\
& f^{\prime \prime \prime}(t)=m(m-1)(m-2) t^{(m-3)} .
\end{aligned}
$$

The $n^{\text {th }}$ repetitive integer differentiation process can therefore be summarized as:

$\frac{d^{n} f}{d t^{n}}=\frac{(m !) t^{(m-n)}}{(m-n) !} \quad$ where $m \geq n$

In a likewise manner, the $n^{\text {th }}$ repetitive integer integration process can also be examined for a power function of time:

$f(t)=t^{m}$.

$\int f(t) d t=\frac{t^{(m+1)}}{m+1}$.

$\iint f(t) d t d t=\frac{t^{(m+2)}}{(m+1)(m+2)}$.

$\iiint f(t) d t d t d t=\frac{t^{(m+3)}}{(m+1)(m+2)(m+3)}$.

$\underbrace{\int \ldots \int f(t) d t \ldots d t}_{n}=\frac{d^{-n} f}{d t^{-n}}=\frac{(m !) t(m+n)}{(m+n) !}$.

Note that a constant of integration can be utilized after the completion of the repetitive integration process. Thus, using either (5) or (10), repetitive differentiation or repetitive integration can be similarly accomplished using either positive or negative values for $n$ respectively.

The gamma function $\Gamma(x)$ can be defined as [10]:

$$
\begin{aligned}
\Gamma(x) & =\lim _{\mu \rightarrow \infty} \frac{\mu^{x}}{x}\left(\frac{1}{x+1}\right)\left(\frac{2}{x+2}\right) \ldots\left(\frac{\mu}{x+\mu}\right) \\
& =\lim _{\mu \rightarrow \infty} \frac{1}{x} \mu^{x} \prod_{j=1}^{\mu}\left(1+\frac{x}{j}\right)^{-1} .
\end{aligned}
$$

Note that the gamma function is not defined for values of $x$ equal to either zero or negative integer values. Furthermore, when $x$ is a positive integer, the gamma function has the following property:

$\Gamma(x)=(x-1) !$ 
Additionally, $\Gamma(x)$ "smoothly connects" the integer values of the factorial function. It is therefore suitable for defining non-integer factorial values. The gamma function is illustrated in Figure 1.

\section{Gamma Function}

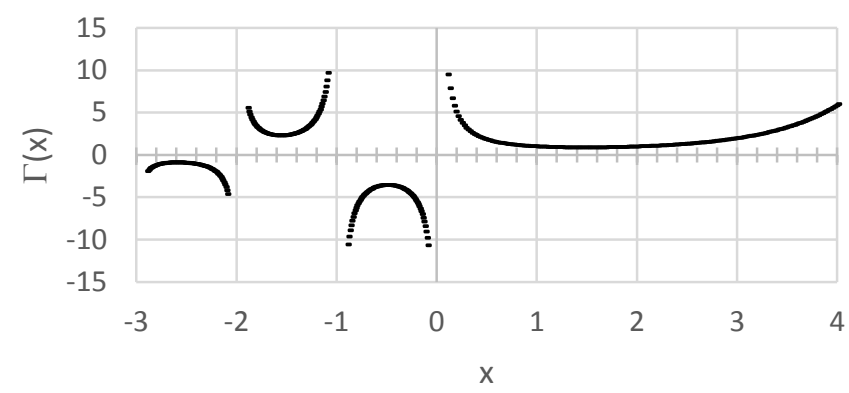

Figure 1. The gamma function is useful in determining noninteger values of the factorial function. It is not defined for zero and negative integer values

Equations (5) and (10) can then be modified to utilize the gamma function:

$\frac{d^{q} f}{d t^{q}}=\frac{\Gamma(m+1) t^{(m-q)}}{\Gamma(m-q+1)}$.

Equation (13) can be used as the definition of the differintegral [11]. Where $q$ can have a positive value; either integer or non-integer. ${ }^{1,2}$ Note that $q$ can also take on integer or non-integer negative values: ${ }^{3}$

$\underbrace{\int \ldots \int f(t) d t \ldots d t}_{q}=\frac{d^{-q} f}{d t^{-q}}=\frac{\Gamma(m+1) t^{(m+q)}}{\Gamma(m+q+1)}$.

The term $q$ is referred to as the order of differintegration [12]. Additionally, $f(t)=\left.\frac{d^{q} f}{d t^{q}}\right|_{q=0}$. As previously stated, $\Gamma(0)$ is not defined. The gamma function is also not defined for negative integer values. Thus, specific fractional derivatives, or fractional integrals, may be unattainable.

Owing to either the positive or negative value of $q$ in (13), the differintegral can therefore be utilized for the fractional differentiation, or fractional integration, of

${ }^{1}$ However, $q$ cannot take on integer values equal to such quantities as: $(m+1)$ or $(m+2)$ or $(m+3)$, etc. power functions. Furthermore, using FC, differentiation and integration may possibly be represented as a continuous process rather than discrete processes.

\subsection{Fractional Calculus and the Taylor Series of an Exponential Function}

The Taylor series for an exponential function is:

$$
\begin{aligned}
e^{-g \cdot t} & =\sum_{n=0}^{N} \frac{(-g \cdot t)^{n}}{n !} \\
& =\frac{(-g \cdot t)^{0}}{0 !}+\frac{(-g \cdot t)^{1}}{1 !}+\frac{(-g \cdot t)^{2}}{2 !} \ldots+\frac{(-g \cdot t)^{N}}{N !} .
\end{aligned}
$$

Thus, for a sufficiently large $N$, an exponential function can be accurately approximated as a summation of power functions. Using the above methodology, the Taylor series for an exponential can therefore be termby-term fractionally differentiated or fractionally integrated:

$$
\frac{d^{q}\left(e^{-g \cdot t}\right)}{d t^{q}}=\sum_{n=0}^{N}\left\{\frac{(-g)^{n}}{n !} \cdot\left[\frac{\Gamma(n+1) t^{(n-q)}}{\Gamma(n-q+1)}\right]\right\} .
$$

For mathematical purposes, $t$ cannot equal zero and be raised to a negative power. However, $t$ can take on positive near-zero values. Negative values of $t$ can also yield complex results. To further reiterate, care must be used when selecting integer values of $q$ to prevent undefined values of the gamma function from occurring.

\section{Examining the Velocity of Aortic Blood Flow}

The esophageal Doppler monitor (EDM) is frequently utilized to assess the velocity of aortic blood flow during systole. The EDM allows clinicians to accurately assess patients' cardiac output and stroke volume during anesthesia and critical care conditions [13]. Figure 2 illustrates this waveform.

This velocity, $v(t)$, can be modelled as [5]:

$v(t)=\alpha \beta e^{-\gamma t}\left(1-\frac{t}{F T}\right) t \quad 0<t<F T$.

${ }^{2}$ Imaginary and complex values of $q$ can also be utilized. However, these will not be addressed in this introductory paper.

${ }^{3}$ Note that an alternative terminology could be that of fractional derivatives and fractional antiderivatives. 
Where $\alpha$ represents an acceleration term and $\beta$ is a dimensionless gain. The time spent in systole is referred to as flow time, $F T$. It should be noted that $\gamma$ can be determined [5]:

$$
\gamma=\frac{2-\left(\frac{F T}{F T p}\right)}{\left(F T_{p}-F T\right)} \quad 0<F T_{p}<F T \text {. }
$$

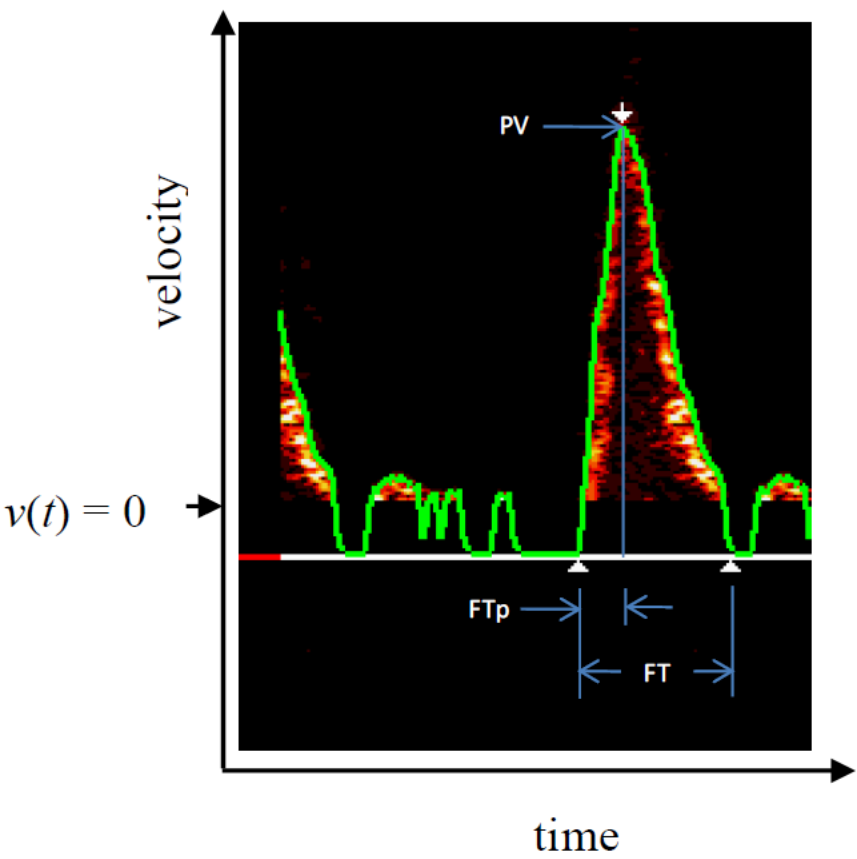

Figure 2. The velocity of aortic blood flow as measured by an EDM. Note that $P V$ represents peak velocity whereas FT signifies the time spent in systole. The time at which $P V$ occurs is referred to as FTp [5]

Note that FTp represents the time at which peak velocity (PV) occurs. This is illustrated in Figure 2. Using a Taylor series, $v(t)$ can subsequently be approximated as a time-based power function:

$$
\begin{aligned}
& v(t)=\alpha \beta\left(\sum_{n=0}^{N} \frac{(-\gamma)^{n} \cdot(t)^{(n+1)}}{n !}-\right. \\
& \left.\frac{1}{F T} \sum_{n=0}^{N} \frac{(-\gamma)^{n} \cdot(t)^{(n+2)}}{n !}\right) \quad 0<t<F T .
\end{aligned}
$$

By means of the aforementioned technique, fractional derivatives and fractional integrals of $v(t)$ can then be determined:

$$
\begin{aligned}
& \frac{d^{q} v}{d t^{q}}=\alpha \beta\left[\sum_{n=0}^{N} \frac{(-\gamma)^{n} \cdot \Gamma(n+2) \cdot(t)^{(n+1-q)}}{n ! \cdot \Gamma(n+2-q)}-\right. \\
& \left.\frac{1}{F T} \sum_{n=0}^{N} \frac{(-\gamma)^{n} \cdot \Gamma(n+3) \cdot(t)^{(n+2-q)}}{n ! \cdot \Gamma(n+3-q)}\right] \quad 0<t<F T
\end{aligned}
$$

Figure 3 demonstrates the continuous differintegral (20) over the range: $-1 \leq q \leq 1$.
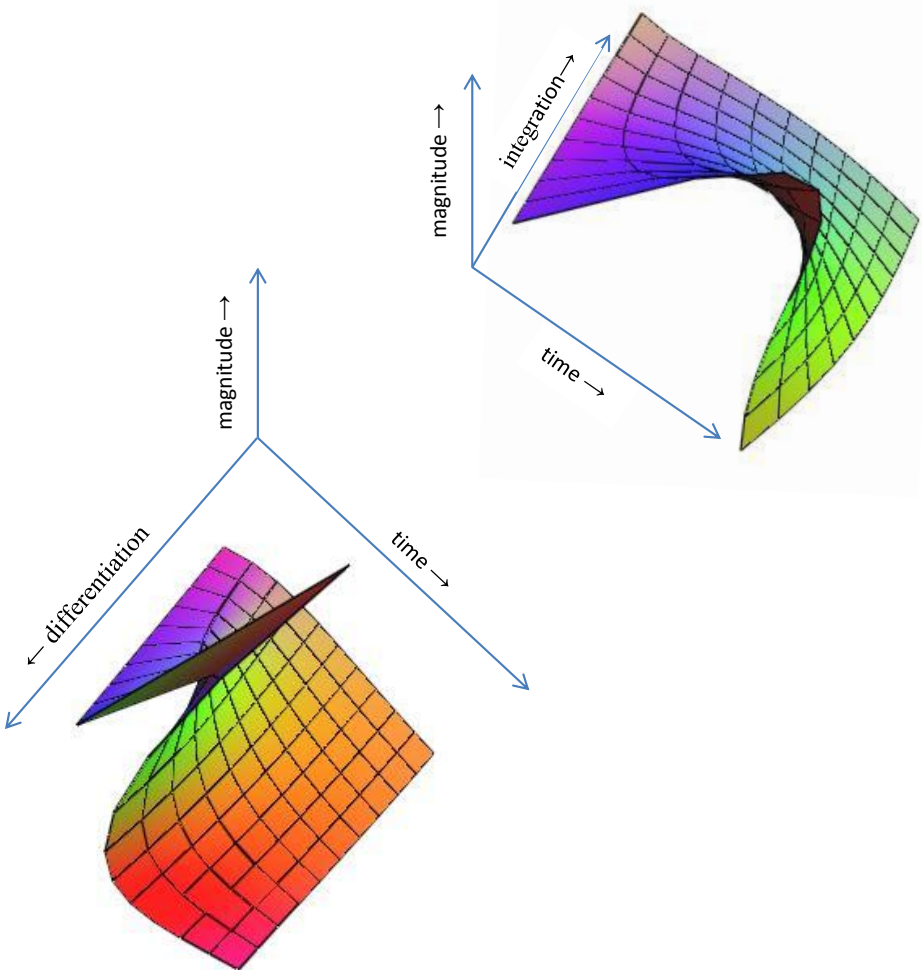

Figure 3. Equation (20), the velocity of aortic blood flow during systole, $v(t)$, represented as a continuous differintegral. Note that fractional integration is associated with $-1<q<0$ whereas fractional differentiation is associated with $0<q<1$. Furthermore, $v(t)=\left.\frac{d^{q} v}{d t^{q}}\right|_{q=0}$

\section{Numerical Assessment}

Using MATHCAD (PTC Corp., Needham, MA, USA) $v(t)$ can be calculated utilizing the numerical values from Table 1. Subsequently, its differintegrals of order -0.7 and 0.1 can both be determined. These functions are illustrated in Figure 4.

Note that the dimension associated with $v(t)$ is $\mathrm{m} / \mathrm{s}$ whereas that of $\frac{d^{(-0.7)} v}{d t^{(-0.7)}}$ is $\mathrm{m} / \mathrm{s}^{(-0.7) .4}$ Furthermore, the dimension of $\frac{d^{(0.1)} v}{d t^{(0.1)}}$ is $\mathrm{m} / \mathrm{s}^{0.1}$.

\footnotetext{
${ }^{4}$ This dimension is equivalent to $\mathrm{m} \cdot \mathrm{s}^{0.7}$.
} 
Table 1. Numerical values used for initial computational purposes

\begin{tabular}{|c|c|c|c|}
\hline Term & Value & Units & Notes \\
\hline$\alpha$ & 7.25 & $\mathrm{~m} / \mathrm{s}^{2}$ & acceleration \\
\hline$\beta$ & 3.00 & dimensionless & gain \\
\hline$\gamma$ & 6.154 & $\mathrm{~s}^{-1}$ & $\begin{array}{c}\text { exponential } \\
\text { decay }\end{array}$ \\
\hline$F T$ & 0.36 & $\mathrm{~s}$ & flow time \\
\hline$F T p$ & 0.1 & $\mathrm{~s}$ & time to peak flow \\
\hline$a$ & -0.7 & dimensionless & $\begin{array}{c}\text { order of } \\
\text { fractional } \\
\text { differintegration }\end{array}$ \\
\hline$b$ & 0.1 & dimensionless & $\begin{array}{c}\text { order of } \\
\text { fractional } \\
\text { differintegration }\end{array}$ \\
\hline
\end{tabular}

\section{The Systolic Pressure-Flow Relationship in the Aorta}

Using (20), a straightforward model of aortic blood pressure, $P(t)$, as a function of the velocity of aortic blood flow during systole is:

$P(t)=\frac{d^{0} P}{d t^{0}}=k \pi r^{2}\left(Z_{a} \frac{d^{a} v}{d t^{a}}+Z_{b} \frac{d^{b} v}{d t^{b}}\right)+C$.

Where $r$ represents the radius of the aorta, and $a$ and $b$ are both velocity-based differintegrals of order -0.7 and 0.1 respectively. The term $Z_{a}$ is "reactance-like" and would be analogous to a combination of elastance and resistance. Whereas $Z_{b}$ would be analogous to a combination of inertia and resistance. Furthermore, $C$ is a constant of integration and $k$ converts units of Pascals to $\mathrm{mmHg}$. In addition:

$\left.\frac{d^{a} v}{d t^{a}}\right|_{t=0}=\left.\frac{d^{b} v}{d t^{b}}\right|_{t=0}=0$

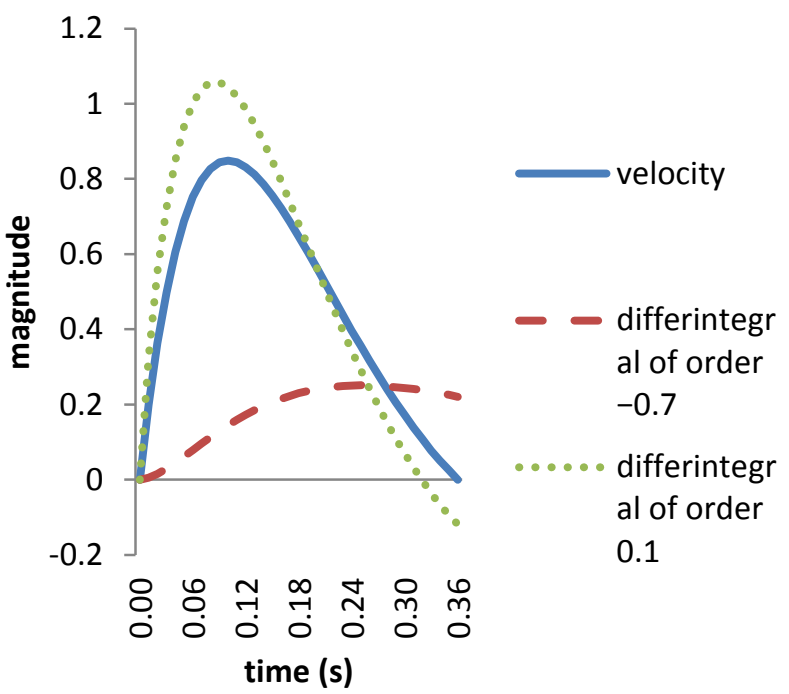

Figure 4. Velocity as a function of time, $v(t)$, and both its associated differintegrals of order -0.7 and 0.1 are displayed

So that $C$ also functions as an initial condition. Moreover, for the purposes of this preliminary assessment, a "trial and error" technique was employed to determine numerical values for $a, b$ and $Z_{a}$ and $Z_{b}$. These are displayed in Table 2 . Note that $Z_{a}$ and $Z_{b}$ have magnitudes which are "ballpark approximate" to those of traditionally-derived resistance, elastance, and inertia.

The above model can also be utilized to assess $\frac{d P}{d t}$ during systole:

$\frac{d P}{d t}=k \pi r^{2}\left(Z_{a} \frac{d^{(a+1)} v}{d t^{(a+1)}}+Z_{b} \frac{d^{(b+1)} v}{d t^{(b+1)}}\right)$.

Both $P(t)$ and $\frac{d P}{d t}$ are illustrated in Figure 5. Note that a positive near-zero initial value for $t$, instead of zero, has to be used in (23) to prevent a "division by zero" singularity error from occurring.

Table 2. Numerical values used for final computational purposes

\begin{tabular}{|c|c|c|c|}
\hline Term & Value & Units & Notes \\
\hline$C$ & 80 & $\mathrm{mmHg}$ & $\begin{array}{c}\text { constant of } \\
\text { integration }\end{array}$ \\
\hline$k$ & 0.0075 & $\mathrm{mmHg} /$ Pascal & $\begin{array}{c}\text { unit } \\
\text { conversion }\end{array}$ \\
\hline$r$ & 0.011 & $\mathrm{~m}$ & aortic radius \\
\hline$Z_{a}$ & $3.157 \cdot 10^{7}$ & $\mathrm{~N} \cdot \mathrm{s}^{a} / \mathrm{m}^{5}$ & $\begin{array}{c}\text { "reactance- } \\
\text { like" term }\end{array}$ \\
\hline$Z_{b}$ & $7.015 \cdot 10^{6}$ & $\mathrm{~N} \cdot \mathrm{s}^{b} / \mathrm{m}^{5}$ & $\begin{array}{c}\text { "reactance- } \\
\text { like" term }\end{array}$ \\
\hline
\end{tabular}




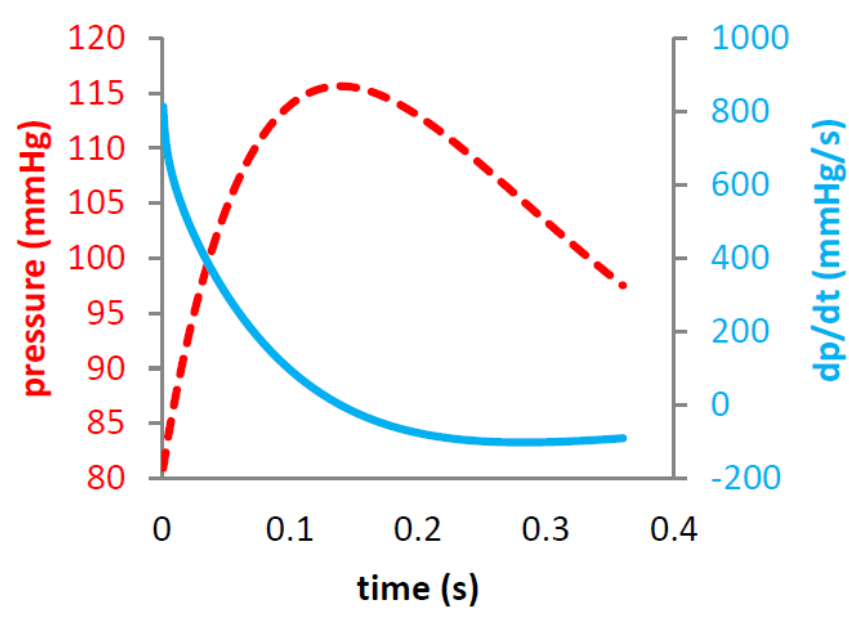

- pressure $(\mathrm{mmHg})=\mathrm{dp} / \mathrm{dt}(\mathrm{mmHg} / \mathrm{s})$

Figure 5. Using fractional calculus, $P(t)$ is modelled using differintegrals which are based upon the velocity of aortic blood flow during systole. Note that $\frac{d P}{d t}$ is also displayed

Straightforward linear algebraic techniques could also be applied. This would allow "real-time" or beat-tobeat assessment of the "reactance-like" terms, $Z_{a}$ and $Z_{b}$. The following matrix relationship is derived using (21):

$k \pi r^{2} \cdot\left[\begin{array}{ll}\left.\frac{d^{a} v}{d t^{a}}\right|_{t=t_{s}} & \left.\frac{d^{b} v}{d t^{b}}\right|_{t=t_{s}} \\ \left.\frac{d^{a} v}{d t^{a}}\right|_{t=t_{e s}} & \left.\frac{d^{b} v}{d t^{b}}\right|_{t=t_{e s}}\end{array}\right] \cdot\left[\begin{array}{c}Z_{a} \\ Z_{b}\end{array}\right]=\left[\begin{array}{c}P_{S} \\ P_{e s}\end{array}\right]$

Thus:

$\left[\begin{array}{c}Z_{a} \\ Z_{b}\end{array}\right]=\frac{1}{k \pi r^{2}}\left\{\left[\begin{array}{ll}\left.\frac{d^{a} v}{d t^{a}}\right|_{t=t_{s}} & \left.\frac{d^{b} v}{d t^{b}}\right|_{t=t_{s}} \\ \left.\frac{d^{a} v}{d t^{a}}\right|_{t=t_{e s}} & \left.\frac{d^{b} v}{d t^{b}}\right|_{t=t_{e s}}\end{array}\right]^{-1} \cdot\left[\begin{array}{c}P_{S} \\ P_{e s}\end{array}\right]\right\}$

\section{Discussion and Conclusion}

Noninvasive assessment, of the hemodynamics within large arteries such as the aorta, has been limited, although invasive means have been commonplace in the clinical setting through catheterization [1], [14]. Advanced imaging modalities can provide geometric and detailed structural changes, but the dynamic properties of the pressure-flow relationship is typically not obtainable. Esophageal Doppler ultrasound measurements, of aortic blood flow velocity, have been demonstrated as a useful minimally-invasive clinical tool [15]. However, central aortic pressure cannot be obtained noninvasively.

Applanation tonometry, applied to peripheral arteries such as the carotid and radial, has been reported for noninvasive monitoring of both pulsatile pressure waveforms and arterial compliance [16]. Subsequently, these recorded peripheral waveforms have also been used to derive central aortic pressure waveforms via a transfer function. Clinically, these have been applied to obtain the augmentation index [17] and to assess vascular stiffness. But the temporal aspects, of the aortic pressure-flow relationship through minimally-invasive means, has yet to be demonstrated.

We have introduced a technique, based on FC, for potentially deriving the temporal relationship of aortic pressure and flow throughout systole. The minimallyinvasive measurement of the velocity of aortic blood flow is accomplished with an EDM; although this can also be obtained with a transthoracic Doppler echocardiographic monitor; which is commonly utilized in many clinics.

Our approach is novel in that the Taylor series expansion of an exponential function was applied to allow term-by-term fractional differentiation and fractional integration. A closely related work using FC was performed by Craiem and Armentaro [18] who examined the power-law stress-strain relationship within sheep aorta; through simultaneous measurements of pulsatile aortic pressure and diameter. They were able to account for a dynamic frequencydependent elastic modulus [19].

The rate of rise of aortic pressure $\frac{d P}{d t}$ and flow velocity $\frac{d v}{d t}$ both have a close relationship with myocardial performance; particularly their peak values, $\max \left(\frac{d P}{d t}\right)$ and $\max \left(\frac{d v}{d t}\right)$ [20]. Thus, the minimallyinvasive assessment of left ventricle contractility is potentially possible using an FC model.

\section{References}

[1] J. K-J. Li, The Arterial Circulation: Physical Principles and Clinical Applications, Totowa, NJ, USA: Humana Press, 2000.

[2] J. K-J. Li, Dynamics of the Vascular System, Singapore: World Scientific Publishing, 2004.

[3] C. Bowman, W-M. Shi, and J. K-J. Li, "A new noninvasive method to obtain blood pressure 
waveforms," Proceedings of the $11^{\text {th }}$ Annual Northeast Bioengineering Conference, 1985.

[4] M. K. Tangella, C. Bowman, J. K-J. Li, "Noninvasive analog measurements of ventricular ejection pressure," Proceedings of the $14^{\text {th }}$ Annual Northeast Bioengineering Conference, pp. 163-165, 1988.

[5] G. Atlas, "Development and application of a logisticbased systolic model for hemodynamic measurements using the esophageal Doppler monitor," Cardiovascular Engineering, vol. 8, no. 3, pp. 159-173, 2008.

[6] M. Dalir, M. Bashour, "Applications of fractional calculus," Applied Mathematical Sciences, vol. 4, no. 21, pp. 1021-1032, 2010.

[7] S. A. David, J. L. Linares, E. M. J. A. Pallone, "Fractional order calculus: historical apologia, basic concepts and some applications," Revista Brasileira de Ensino de Física, vol. 33, no. 4, pp. 4302-1-4302-7, 2011.

[8] D. J. Djordjevic, J. Jaric, B. Fabry, J. J. Fredberg, D. Stamenovic, "Fractional derivatives embody essential features of cell rheological behavior," Annals of Biomedical Engineering, vol. 31, pp. 692699, 2003.

[9] S. Das, K. Moharatna, "Fractional dynamical model for the generation of ECG like signals from filtered coupled Van-der Pol oscillators," Computer Methods and Programs in Biomedicine, vol. 112, pp. 490-507, 2013.

[10] J. Bonnar, The Gamma Function, Applied Research Press, 2013.

[11] S. Das, Functional Fractional Calculus, Heidelberg, Germany: Springer-Verlag, 2011.

[12] L. M. B. C. Campos, "On a generalized Mittag-Leffler theorem and implicit differintegration," Society for Industrial and Applied Mathematics (SIAM): Journal on Mathematical Analysis, vol. 20, no. 2, pp. 454-467, 1989.

[13] G. Atlas, D. Brealey, S. Dhar, G. Dikta, M. Singer, "Additional hemodynamic measurements with an esophageal Doppler monitor: a preliminary report of compliance, force, kinetic energy, and afterload in the clinical setting," Journal of Clinical Monitoring and Computing, vol. 26, no. 6, pp. 473482, 2012.

[14] J. K-J. Li, A. G. W. Van Brummelen, A. Noordergraaf, "Fluid-filled blood-pressure measurement systems," Journal of Applied Physiology, vol. 40, pp. 839- 843, 1976.
[15] G. Atlas, J. K-J. Li, and J. B. Kostis, "A comparison of mathematical models of left ventricular contractility derived from aortic blood flow velocity and acceleration: application to the esophageal Doppler monitor," Biomedical Engineering Letters, vol. 4, pp. 301-315, 2014.

[16] G. Drzewiecki, V. Bansal, J. K-J. Li, "Noninvasive measurement of single artery pressure and compliance using tonometry," Conference Proceedings Association for the Advancement of Medical Instrumentation, vol. 30, 1996.

[17] J. E. Sharman, J. E. Davies, C. Jenkins, T. H. Marwick, "Augmentation index, left ventricular contractility, and wave reflection," Hypertension, vol. 54 pp. 1099-1105, 2009.

[18] D.O. Craiem, R. L. Armentano, "Arterial viscoelasticity: a fractional derivative model," Conference Proceedings IEEE Engineering in Medicine and Biology Society, vol. 1, pp. 1098-1101, 2006.

[19] J. K-J. Li, J. Melbin, A. Riffle, A. Noordergraaf, "Pulse Wave Propagation," Circulation Research, vol. 49, pp. 442-452, 1981.

[20] J. K-J. Li, H. Yamazaki, M. M. Bodenheimer, V. S. Banka, R. H. Helfant, "Myocardial performance assessed by peak derivatives of peripheral pressure and flow pulses," Federation Proceedings, vol. 38, pp. 1449, 1979. 